\title{
A Millimeter-Wave CMOS LC-Tank VCO With an Admittance-Transforming Technique
}

\author{
Hsieh-Hung Hsieh, Student Member, IEEE, Yu-Hsin Chen, Member, IEEE, and Liang-Hung Lu, Member, IEEE
}

\begin{abstract}
A novel circuit topology suitable for millimeter-wave voltage-controlled oscillators (VCOs) is presented. With the admittance-transforming technique, the proposed VCO can operate at a frequency close to the $f_{\max }$ of the transistors while maintaining remarkable circuit performance in terms of phase noise, tuning range, and output power. Using a standard micrometer CMOS process, a $U$-band VCO is implemented for demonstration. The fabricated circuit exhibits a frequency tuning range of $1.1 \mathrm{GHz}$ in the vicinity of $50 \mathrm{GHz}$. The measured output power and phase noise at $1-\mathrm{MHz}$ offset are $-11 \mathrm{dBm}$ and $-101 \mathrm{dBc} / \mathrm{Hz}$, respectively. Operated at a supply voltage of $1.8 \mathrm{~V}$, the VCO core consumes a dc power of $45 \mathrm{~mW}$.
\end{abstract}

Index Terms-Admittance transforming, coplanar waveguide (CPW), $L C$-tank oscillators, millimeter wave, phase noise, quality (Q) factor, voltage-controlled oscillators (VCOs).

\section{INTRODUCTION}

$\mathbf{R}$ ECENTLY, with the emerging wireless applications such as the broadband wireless local area network (WLAN) at $60 \mathrm{GHz}$, the automatic cruise control at 76-77 GHz, and the advanced image sensors at $94 \mathrm{GHz}$, the development of low-cost and high-performance monolithic microwave integrated circuits have attracted great attention. Due to the unparalleled advantages in both fabrication cost and high-level integration, CMOS technology appears to be well suited for the circuit implementations. Unfortunately, the restricted cutoff frequency $f_{T}$ and maximum oscillation frequency $f_{\max }$ have impeded the evolution of RF frontends for millimeter-wave applications. In order to enhance the high-frequency performance of the CMOS RF circuits, various design strategies have been proposed over the past few years [1]-[3].

Being a crucial component in the RF frontends, the voltagecontrolled oscillator (VCO) provides the required local oscillator (LO) signal for frequency conversion. Conventionally, the cross-coupled $L C$-tank topology is widely used for high-frequency designs [4]-[9]. However, as the operating frequency approaches to the $f_{\max }$ of the active devices, the realization of the $L C$-tank CMOS VCOs encounters great challenges due

Manuscript received February 9, 2007; revised May 5, 2007. This work was supported by the MTK Education Foundation. This work was supported in part by the National Science Council under Grant 94-2220-E-002-026 and Grant 94-2220-E-002-009.

H.-H. Hsieh and L.-H. Lu are with the Department of Electrical Engineering and Graduate Institute of Electronics Engineering, National Taiwan University, Taipei 10617, Taiwan, R.O.C. (e-mail: lhlu@cc.ee.ntu.edu.tw).

Y.-H. Chen is with the Graduate Institute of Communication Engineering, National Taiwan University, Taipei 10617, Taiwan, R.O.C.

Digital Object Identifier 10.1109/TMTT.2007.903347

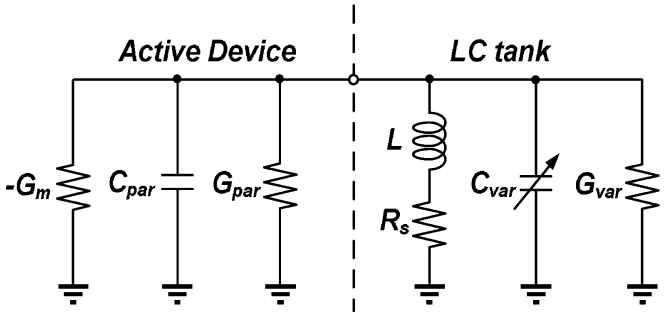

(a)

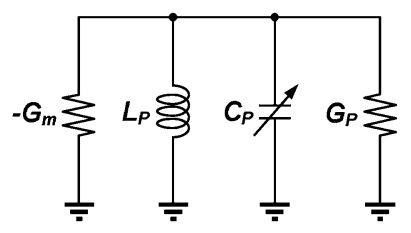

(b)

Fig. 1. (a) Equivalent half circuit of the conventional $L C$-tank VCO. (b) Simplified model for circuit analysis.

to the inherently low quality $(Q)$ factors of the on-chip passive elements, making it extremely difficult to meet the stringent startup conditions for sustained oscillation. In order to alleviate the limitations on the operating frequencies, an admittance-transforming technique is presented. By inserting inductive elements in series connection with the varactor, the equivalent shunt conductance of the tank can be effectively reduced at higher frequencies, leading to a VCO design close to the $f_{\max }$ of the MOSFETs. In addition, with the nature of the tunable effective inductance, a reasonable frequency tuning range is also demonstrated. Using a standard 0.18- $\mu \mathrm{m}$ CMOS process, a VCO prototype is successfully implemented at the $50-\mathrm{GHz}$ frequency band.

The remainder of this paper is organized as follows. In Section II, the concept of the admittance-transforming technique is illustrated. The proposed VCO topology and the circuit implementations are presented in Sections III and IV, respectively. The experimental results are provided in Section V. Finally, a conclusion is given in Section VI.

\section{AdMITTANCE-TRANSFORMING TECHNIQUE}

Fig. 1(a) shows the equivalent half circuit of the conventional $L C$-tank VCO where the resonator is composed of an inductor $L$ and a varactor $C_{\mathrm{var}}$. The active devices are employed to provide a negative conductance $-G_{m}$, compensating for the losses from the passive elements. For the derivations of the oscillation frequency and the startup conditions, a simplified equivalent circuit, as shown in Fig. 1(b), is employed. According to 
the narrowband approximation, the shunt components including $L_{P}, C_{P}$, and $G_{P}$ can be estimated as

$$
\begin{aligned}
& L_{P}=L \\
& C_{P}=C_{\mathrm{var}}+C_{\mathrm{par}} \\
& G_{P} \approx G_{\mathrm{var}}+G_{\mathrm{par}}+R_{s} /(\omega L)^{2} .
\end{aligned}
$$

The oscillation frequency is given by

$$
f_{0}=\frac{1}{2 \pi \sqrt{L_{P} C_{P}}}
$$

and the startup condition yields

$$
G_{m}>G_{P}
$$

Typically, $G_{P}$ is dominated by the loss of the on-chip inductors for conventional $L C$-tank CMOS VCO designs at an oscillation frequency below $10 \mathrm{GHz}$. However, for applications at millimeter-wave frequencies, $G_{P}$ is mainly determined by the losses from the varactor, imposing a fundamental limitation on the maximum operating frequency of the $L C$-tank VCOs [4].

To alleviate the frequency limitations due to presence of the varactors, an admittance-transforming technique is proposed. A conceptual illustration of this technique is shown in Fig. 2(a). With a series connection of the varactor $C_{\mathrm{var}}$ and an additional inductor $L_{1}$, the input admittance of $Y_{\text {in }}$ can be expressed as

$$
Y_{\mathrm{in}}=\frac{1}{j \omega L_{1}+\left(G_{\mathrm{var}}+j \omega C_{\mathrm{var}}\right)^{-1}}=G_{T}+\left(j \omega L_{T}\right)^{-1}
$$

where

$$
\begin{aligned}
G_{T} & =\frac{G_{\mathrm{var}}}{L_{1}^{2} C_{\mathrm{var}}^{2} \omega^{4}+\left(L_{1}^{2} G_{\mathrm{var}}^{2}-2 L_{1} C_{\mathrm{var}}\right) \omega^{2}+1} \\
L_{T} & =\left(1-\frac{L_{1} C_{\mathrm{var}} \omega^{2}-1}{L_{1}^{2} C_{\mathrm{var}}^{2} \omega^{4}+\left(L_{1}^{2} G_{\mathrm{var}}^{2}-L_{1} C_{\mathrm{var}}\right) \omega^{2}}\right) L_{1} .
\end{aligned}
$$

As indicated in (7), $G_{T}$ is smaller than $G_{\text {var }}$ provided that

$$
\omega>\sqrt{\frac{2}{L_{1} C_{\mathrm{var}}} \cdot \frac{Q_{\mathrm{var}}^{2}}{Q_{\mathrm{var}}^{2}+1}} \approx \frac{\sqrt{2}}{\sqrt{L_{1} C_{\mathrm{var}}}}=\sqrt{2} \omega_{R}
$$

where $Q_{\mathrm{var}}$ is the $Q$ factor of the varactor and $\omega_{R}$ is the resonant frequency of $L_{1}$ and $C_{\mathrm{var}}$. With proper choice in the value of the inductance $L_{1}$, the high-frequency losses from the varactors can be effectively reduced by the admittance-transforming technique. Moreover, as the value of $C_{\mathrm{var}}$ varies, the imaginary part of $Y_{\text {in }}$ can be treated as a tunable inductance $L_{T}$ at a frequency higher than $\omega_{R}$, enabling the frequency tuning mechanism for VCO operations. Note that, in order to simplify the analysis, the effective series resistance of $L_{1}$ is neglected in the above derivations.

To further investigate the influence of the loss from $L_{1}$, a series resistance $R_{s 1}$ is included in the equivalent circuit, as shown in Fig. 2(b), and the input admittance $Y \prime_{\text {in }}$ is given by

$$
Y_{\text {in }}^{\prime}=G_{T}^{\prime}+\left(j \omega L_{T}^{\prime}\right)^{-1} .
$$

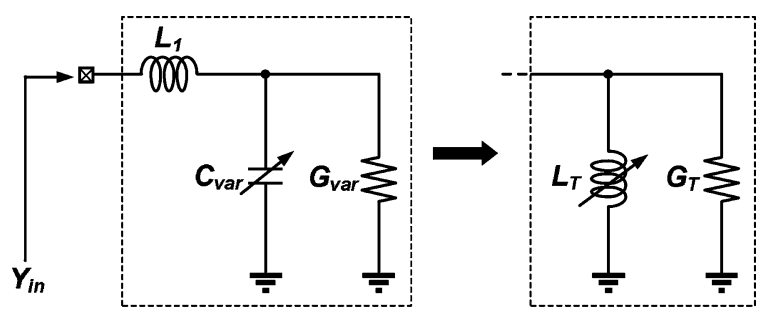

(a)

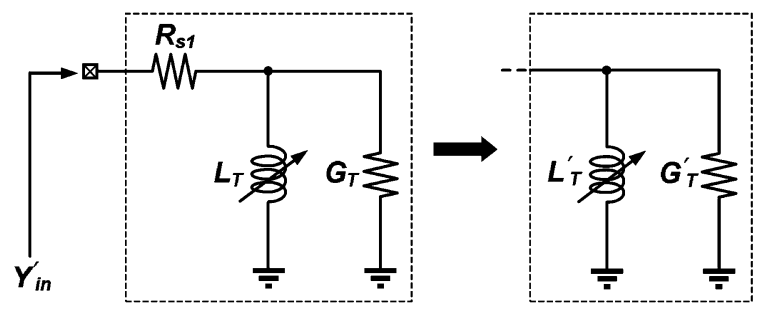

(b)

Fig. 2. Equivalent circuits of the series $L C$-resonator for the admittance-transforming technique with: (a) lossless and (b) lossy inductor $L_{1}$.

Provided $R_{s 1} G_{T} \ll 1$ and $\left(\omega L_{T} / R_{s 1}\right)^{2} \gg 1$ in typical design cases, $G \prime_{T}$ and $L \prime_{T}$ in (10) are approximated by

$$
\begin{aligned}
G_{T}^{\prime} & \approx G_{T}+\frac{1}{Q_{L 1} \omega L_{1}} \cdot\left(\frac{L_{1}}{L_{T}}\right)^{2} \\
L_{T}^{\prime} & \approx L_{T}
\end{aligned}
$$

where $Q_{L 1}$ is the $Q$ factor of the inductor $L_{1}$. From (11), it is apparent that the value of $G_{T}$ depends on $R_{s 1}$. Fortunately, the second term in (11) is typically smaller than $G_{\mathrm{var}}$ by one order of magnitude at millimeter-wave frequencies. As a result, the proposed admittance-transforming technique is still effective with the inductor losses taken into account.

In order to have a better understanding of the admittancetransforming technique, simulations on the series resonator is performed by taking the parasitics and losses into consideration. For a $0.2-\mathrm{pF}$ varactor with various values of inductance $L_{1}$, the resulting $G_{T}$ is shown in Fig. 3(a). As predicted in (7), the shunt conductance can be effectively reduced at higher frequencies. On the other hand, with a fixed $L_{1}$ of $0.25 \mathrm{nH}$, the equivalent inductance $L_{T}$ is demonstrated in Fig 3(b) as the value of $C_{\mathrm{var}}$ varies, featuring a frequency tuning mechanism for the $\mathrm{VCO}$ circuit.

\section{Proposed Topology of VCO}

Fig. 4 shows the schematic of the proposed VCO, where the cross-coupled pair $M_{1}$ and $M_{2}$ is used to provide the required negative conductance and the resonator is established by the inductor $L_{2}$ and the varactor $C_{\mathrm{var}}$. In consideration of the flicker noise contributed by the active devices, a pMOS transistor is employed as the tail current such that lower phase noise can be achieved. Meanwhile, the proposed admittance-transforming technique is introduced by inserting the inductors $L_{1}$ in the series path with the varactors. As a result, the $\mathrm{VCO}$ can operate at 


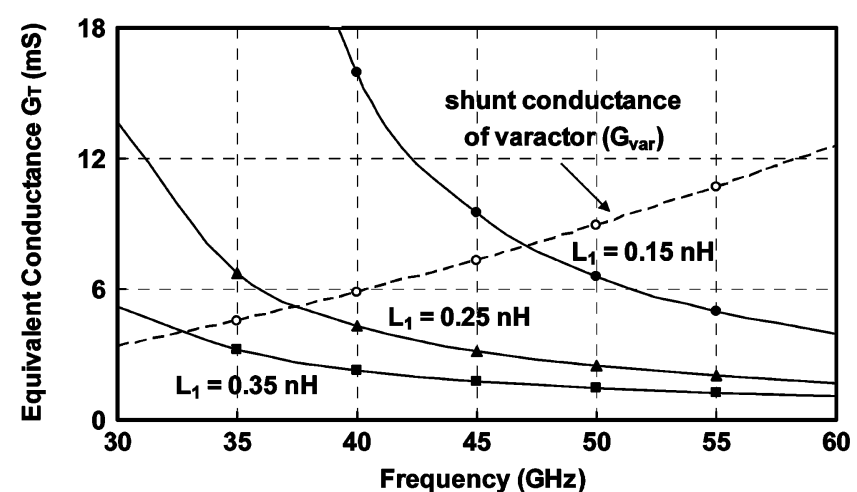

(a)

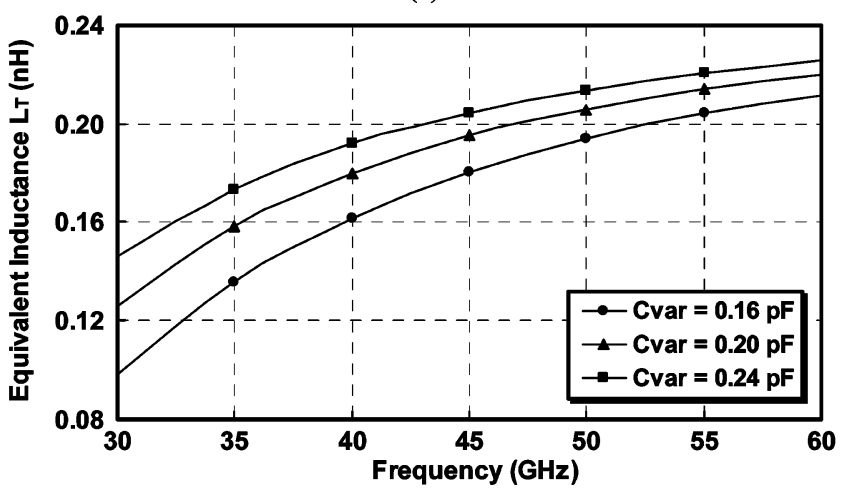

(b)

Fig. 3. (a) Simulated equivalent conductance $G_{T}$ for various values of $L_{1}$ with $C_{\text {var }}=0.2 \mathrm{pF}$. (b) Simulated equivalent inductance $L_{T}$ for various values of $C_{\text {var }}$ with $L_{1}=0.25 \mathrm{nH}$.

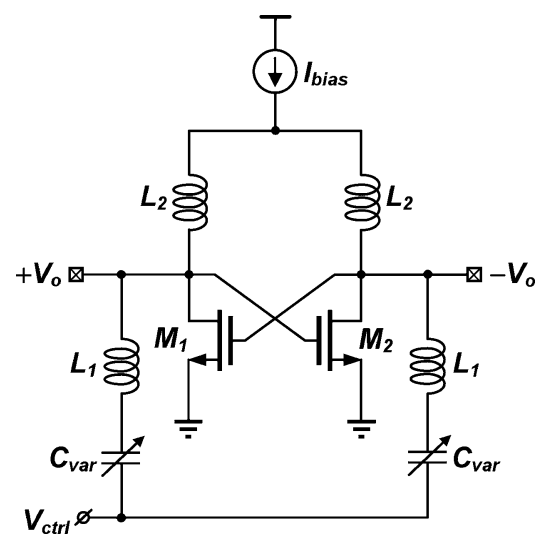

Fig. 4. Circuit topology of the proposed millimeter-wave VCO.

much higher frequencies than the conventional ones while maintaining desirable circuit performance. To gain more insight into the proposed VCO topology, detailed circuit analysis is given as follows.

\section{A. Startup Condition and Oscillation Frequency}

For the derivations of the oscillation frequency and the startup conditions, the equivalent half circuit of the proposed VCO is shown in Fig. 5, where $G_{2}$ and $G_{\text {par }}$ represent the losses from the on-chip inductor $L_{2}$ and the active devices, respectively. Note that the loss of the inductor is typically modeled by a series resistance. In the equivalent-circuit presentation, the nar-

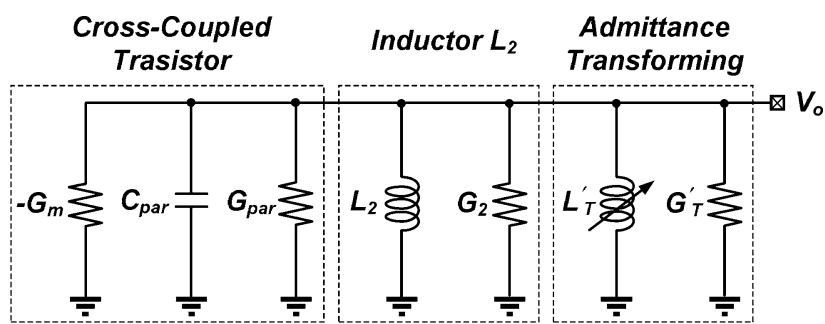

Fig. 5. Equivalent half circuit of proposed VCO.

rowband approximation is employed, and the shunt conductance $G_{2}$ is given by

$$
G_{2} \approx R_{s 2} / \omega^{2} L_{2}^{2}
$$

where $R_{s 2}$ is the equivalent series resistance of $L_{2}$. As for the inductance $L I_{T}$ and shunt conductance $G \prime_{T}$, the analytic expressions in terms of $L_{1}$ and $C_{\text {var }}$ are given in (11) and (12), respectively. To initiate the oscillation, the negative conductance should be sufficiently large to cancel out the total parallel conductance

$$
G_{m}>G_{T}^{\prime}+G_{\mathrm{par}}+G_{2}
$$

On the other hand, the oscillation frequency is given by

$$
f_{0}=\frac{1}{2 \pi \sqrt{\left(L_{2} / / L_{T}^{\prime}\right) \cdot C_{\mathrm{par}}}} .
$$

From (14) and (15), it is apparent that the use of the admittance-transforming technique leads to an alleviated startup condition and a higher oscillation frequency compared with the conventional circuit topology. In addition, by varying the capacitance of the varactor from $C_{\mathrm{var}, \min }$ to $C_{\mathrm{var}, \max }$ through the controlled voltage, the tuning range of the $\mathrm{VCO}$ is defined by

$$
\begin{aligned}
\Delta f_{0}=\frac{1}{2 \pi \sqrt{C_{\mathrm{par}}\left(L_{2} / / L_{T, \min }^{\prime}\right)}} \\
\cdot\left(1-\sqrt{\left.\frac{L_{T, \min }^{\prime}}{L_{T, \max }^{\prime}} \cdot \frac{L_{2}+L_{T, \max }^{\prime}}{L_{2}+L_{T, \min }^{\prime}}\right) .}\right.
\end{aligned}
$$

Note that $L \prime_{T, \text { max }}$ and $L \prime_{T, \text { min }}$ are the maximum and minimum values of the tunable inductance $L I_{T}$, respectively, which can be calculated by (17) and (18), shown at the bottom of the following page. From (16)-(18), it is clear that the tuning range in this study is related to the values of $L_{1}, L_{2}$, and $C_{\mathrm{var}}$. In order to evaluate the tuning characteristics, the simulated tuning range of the proposed VCO as a function of the inductance $L_{1}$ is shown in Fig. 6(a), where the value of $L_{2}$ is determined by $L_{1}$ such that the central oscillation frequency of the VCO remains unchanged. Based on the simulation results, the tuning range degrades as the value of $L_{1}$ increases. Meanwhile, the transconductance required for the VCO startup is effectively reduced. A design tradeoff is thus provided between the startup condition and the tuning range for the circuit implementations.

One interesting phenomenon appearing in Fig. 6(a) is that the tuning range increases as the size of the varactor decreases. 


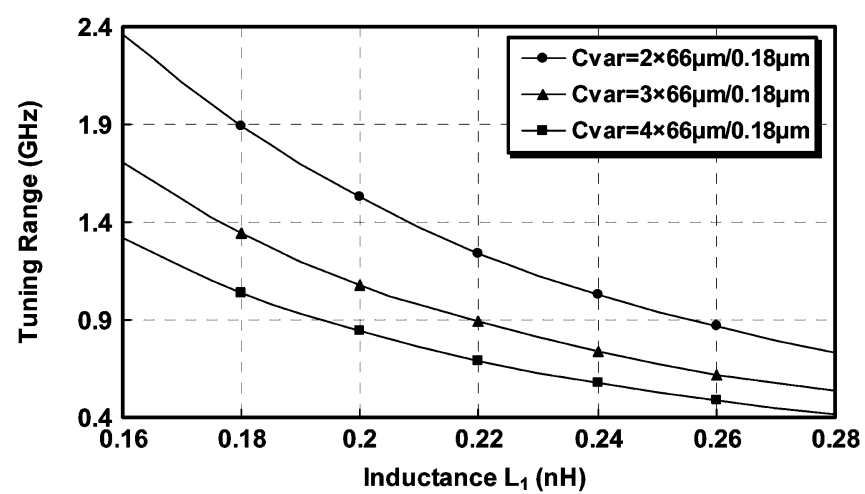

(a)

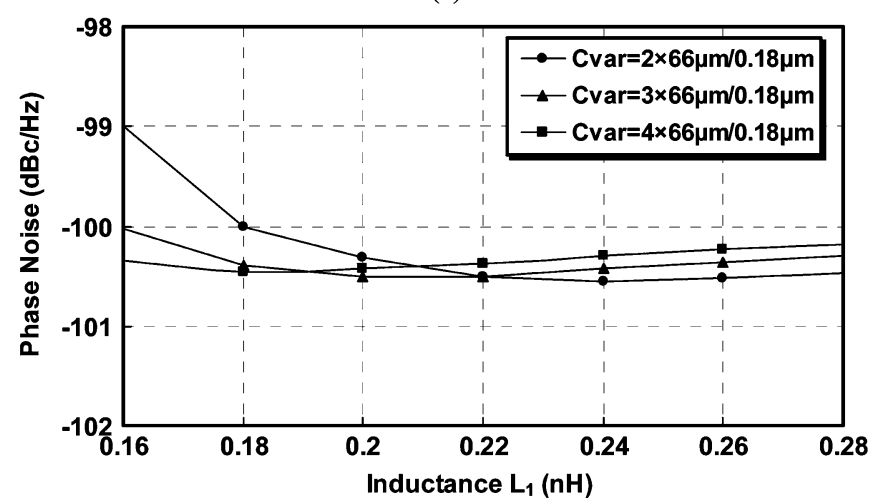

(b)

Fig. 6. Simulated: (a) tuning range and (b) phase noise of the $50-\mathrm{GHz} \mathrm{VCO}$ as a function of $L_{1}$ for various $C_{\text {var values. }}$

This observation conflicts with the design concept in conventional VCO circuits. By employing the admittance-transforming technique, the capacitance of the varactor is effectively transferred into a tunable inductance at the frequency of interest. Consequently, an enhanced tuning range can be achieved by decreasing the size of the varactor. However, for a given operating frequency, (9) indicates that the inductance $L_{1}$ is inversely proportional to the value of $C_{\mathrm{var}}$, setting a lower bound for the varactor size in a practical design.

Another important feature of the admittance-transforming technique is the relation between the frequency tuning range and the phase noise. For conventional VCO circuits, the tuning range increases with the varactor size. As the $Q$ factor of the varactors degrades significantly with the device size, especially at millimeter-wave frequencies, the wide tuning-range VCOs usually suffer from an inferior phase noise. To evaluate the noise performance of the proposed $\mathrm{VCO}$, circuit simulations are performed with identical design parameters in the investigation of the tuning range. The simulated phase noise is shown in Fig. 6(b). By employing the admittance-transforming technique, the shunt conductance of the varactor is suppressed, while the frequency tuning is achieved by the tunable effective inductance. As indicated in the simulation results, the VCO exhibits a phase noise virtually independent of the tuning range, which is a unique and desirable property for the circuit optimization.

\section{B. Phase Noise}

According to the Leeson's model [10], the phase noise of the proposed VCO in the $1 / f^{2}$ region can be expressed as

$$
L\{\Delta f\}=10 \log \left\{\frac{2 F k T}{P_{\text {sig }}}\left[1+\left(\frac{f_{0}}{2 Q \Delta f}\right)^{2}\right]\right\}
$$

where $F$ is the excess noise factor, $Q$ is the $Q$ factor of the $L C$-tank, and $\Delta f$ is the frequency offset. As indicated in (19), the phase noise is strongly influenced by the $Q$ factor of the tank, which can be expressed as

$$
Q=\frac{1}{\left(G_{T}^{\prime}+G_{\mathrm{par}}+G_{2}\right)} \sqrt{\frac{C_{\mathrm{par}}}{L_{2} / / L_{T}^{\prime}}} .
$$

As indicated in (20), reduced phase noise can be achieved in the VCO design by suppressing the shunt conductance $\left(G_{T}+\right.$ $\left.G_{\mathrm{par}}+G_{2}\right)$ and by increasing the value of $C_{\mathrm{par}} /\left(L_{2} / / L \prime_{T}\right)$. However, an increasing ratio of $C_{\mathrm{par}} /\left(L_{2} / / L_{T}\right)$ requires large cross-coupled transistors, which simultaneously increases the shunt conductance $G_{\mathrm{par}}$ and possibly the phase noise. Therefore, the values of $L_{1}, L_{2}$, and $C_{\mathrm{par}}$ should be chosen carefully in the proposed VCO for optimum phase-noise performance.

Fig. 7 shows the simulated phase noise of the VCO at $1-\mathrm{MHz}$ offset with a fixed oscillation frequency of $50 \mathrm{GHz}$ and a varactor value of $0.2 \mathrm{pF}$. As the values of $L_{1}$ and $L_{2}$ increase, the phase noise tends to decrease due to the smaller $C_{\text {par }}$ required. However, further increase in $L_{1}$ and $L_{2}$ leads to higher phase noise due to the degraded $Q$ factors of the inductors. In order to achieve the lowest phase noise possible, the $Q$ factors of the varactors and inductors have to be taken into consideration. In this particular example, the optimum phase noise is approximately $-100 \mathrm{dBc} / \mathrm{Hz}$ provided that $L_{1}$ and $L_{2}$ are 0.25 and $0.2 \mathrm{nH}$, respectively, with a $Q$ factor of 10 .

\section{Output Buffers}

In order to drive the $50-\Omega$ impedance of the test instrument, an output buffer is required for the circuit design. For a VCO

$$
\begin{aligned}
& L_{T, \text { max }}^{\prime}=\left(1-\frac{L_{1} C_{\mathrm{var}, \text { max }} \omega^{2}-1}{L_{1}^{2} C_{\mathrm{var}, \text { max }}^{2} \omega^{4}+\left(L_{1}^{2} G_{\mathrm{var}, \text { max }}^{2}-L_{1} C_{\mathrm{var}, \text { max }}\right) \omega^{2}}\right) L_{1} \\
& L_{T, \text { min }}^{\prime}=\left(1-\frac{L_{1} C_{\mathrm{var}, \text { min }} \omega^{2}-1}{L_{1}^{2} C_{\mathrm{var}, \text { min }}^{2} \omega^{4}+\left(L_{1}^{2} G_{\mathrm{var}, \text { min }}^{2}-L_{1} C_{\mathrm{var}, \text { min }}\right) \omega^{2}}\right) L_{1}
\end{aligned}
$$




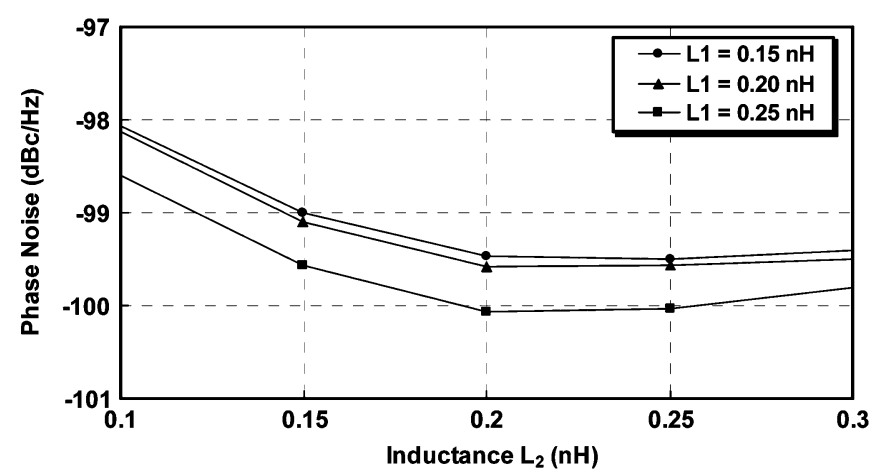

Fig. 7. Simulated phase noise of the proposed VCO with an oscillation frequency of $50 \mathrm{GHz}$ and a varactor of $0.2 \mathrm{pF}$.

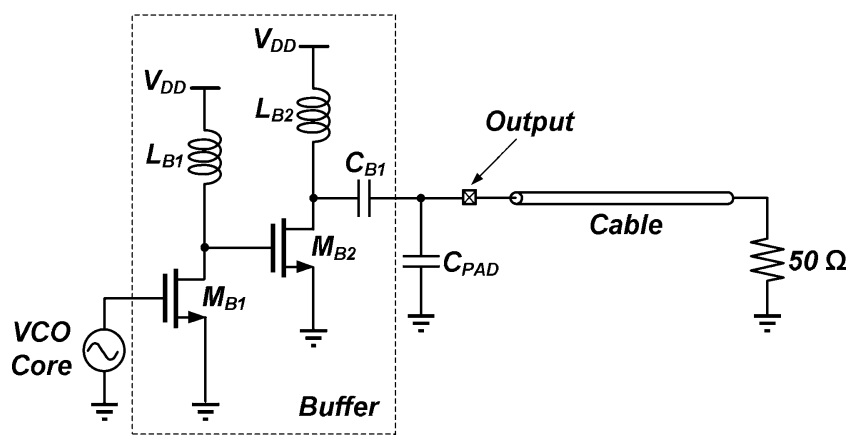

Fig. 8. Circuit schematic of the output buffer.

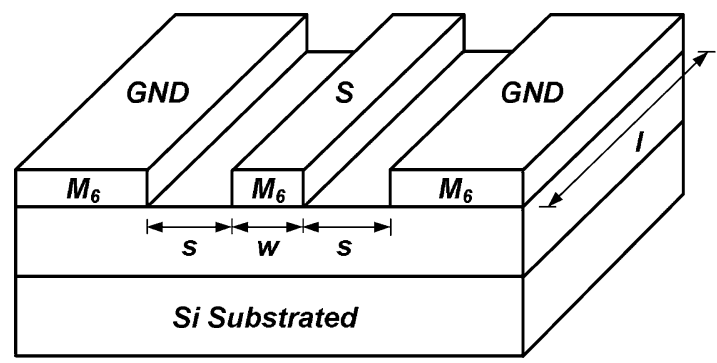

Fig. 9. Structure of the CPW.

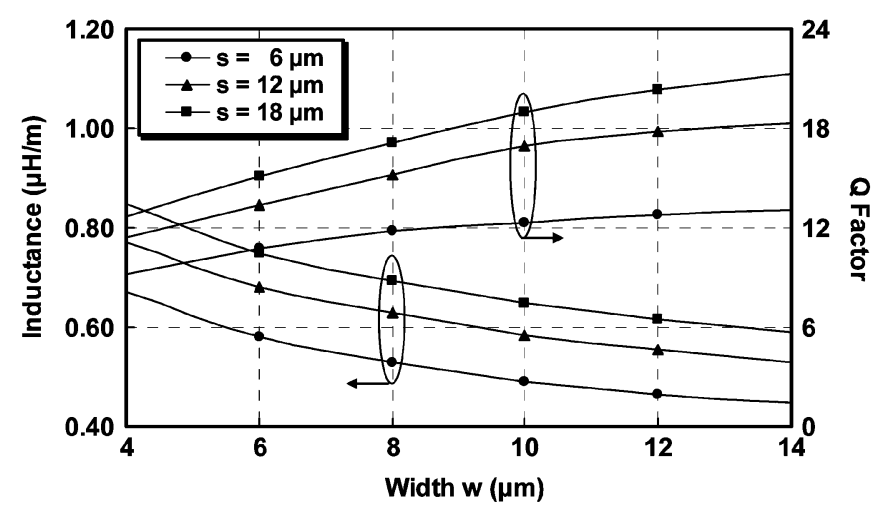

Fig. 10. Extracted equivalent inductance and $Q$ factor of the $\mathrm{CPW}$ with various design parameters.

operating at a frequency close to the $f_{\max }$ of the transistors, the output buffer is also a great design challenge. In this study, a

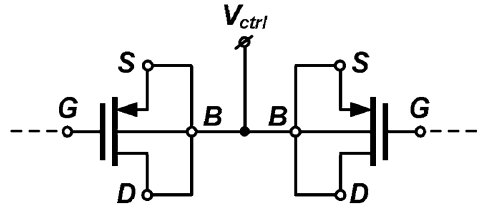

(a)

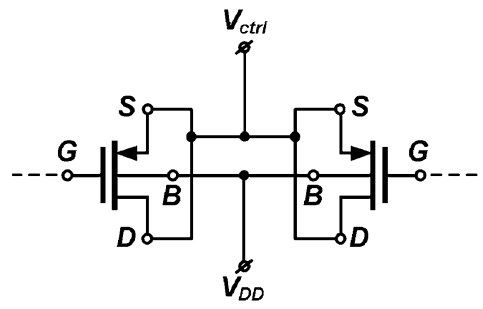

(b)

Fig. 11. (a) Conventional and (b) inversion-mode pMOS varactors.

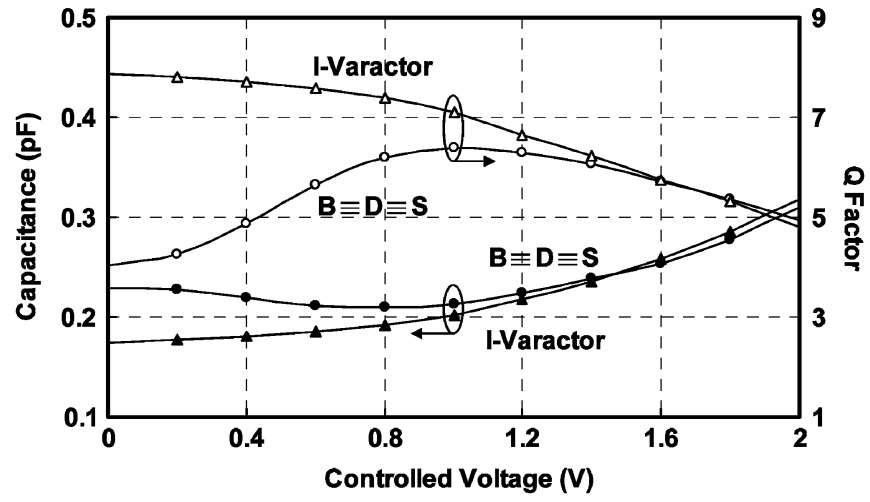

Fig. 12. Simulated capacitance and $Q$ factor of the conventional and the inversion-mode varactor.

TABLE I

Circuit PARAmeters OF THE $U$-BAND VCO

\begin{tabular}{|c|c|c|}
\hline \multicolumn{2}{|c|}{ Devices } & \multicolumn{2}{c|}{ Design Values } \\
\hline \multicolumn{2}{|c|}{$M_{l}, M_{2}$} & \multicolumn{2}{c|}{$2.5 \times 14 \mu \mathrm{m} / 0.18 \mu \mathrm{m}$} \\
\hline \multirow{2}{*}{ Buffers } & $M_{B 1}$ & \multicolumn{2}{|c|}{$2.5 \times 3 \mu \mathrm{m} / 0.18 \mu \mathrm{m}$} \\
\cline { 2 - 3 } & $M_{B 2}$ & \multicolumn{2}{|c|}{$2.5 \times 4 \mu \mathrm{m} / 0.18 \mu \mathrm{m}$} \\
\hline \multirow{2}{*}{$L_{1}$} & Inductance & $0.24 \mathrm{nH}$ \\
\cline { 2 - 3 } & Q Factor & 11.0 \\
\hline \multirow{2}{*}{$L_{2}$} & Inductance & $0.09 \mathrm{nH}$ \\
\cline { 2 - 3 } & Q Factor & 14.0 \\
\hline \multirow{2}{*}{$C_{v a r}$} & Capacitance & $0.17 \sim 0.32 \mathrm{pF}$ \\
\cline { 2 - 3 } & Q Factor & $4.8 \sim 7.8$ \\
\hline \multirow{2}{*}{$L_{B I}$} & Inductance & $0.26 \mathrm{nH}$ \\
\cline { 2 - 3 } & Q Factor & 8.2 \\
\hline \multirow{2}{*}{$L_{B 2}$} & Inductance & $0.37 \mathrm{nH}$ \\
\cline { 2 - 3 } & Q Factor & 6.5 \\
\hline \multirow{2}{*}{$C_{B 1}$} & Capacitance & $0.8 \mathrm{pF}$ \\
\cline { 2 - 3 } & Q Factor & 5.6 \\
\hline \multirow{2}{*}{} & & \\
\hline
\end{tabular}

two-stage buffer with common-source MOSFETs is employed [4], [11]. Fig. 8 shows the circuit schematic where $L_{B 1}, L_{B 2}$, and $C_{B 1}$ are on-chip passive components. The transistor sizes and inductance values are chosen such that the loading effect on 


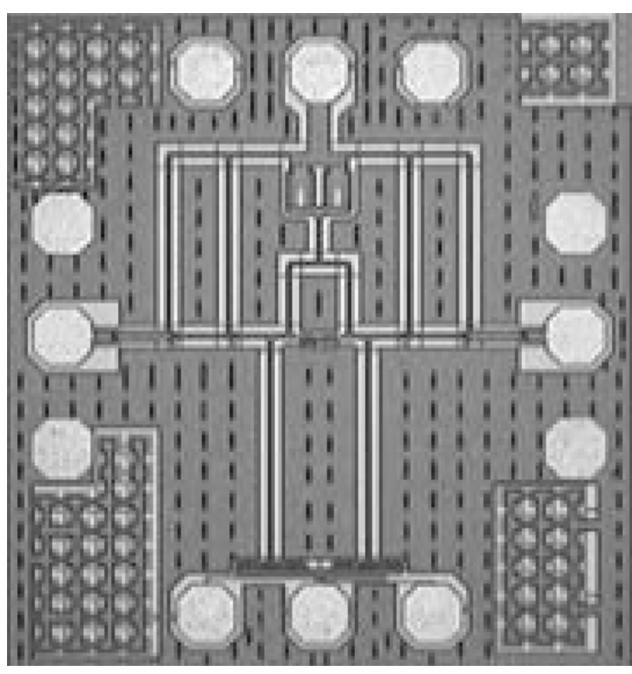

Fig. 13. Microphotograph of the fabricated circuit.

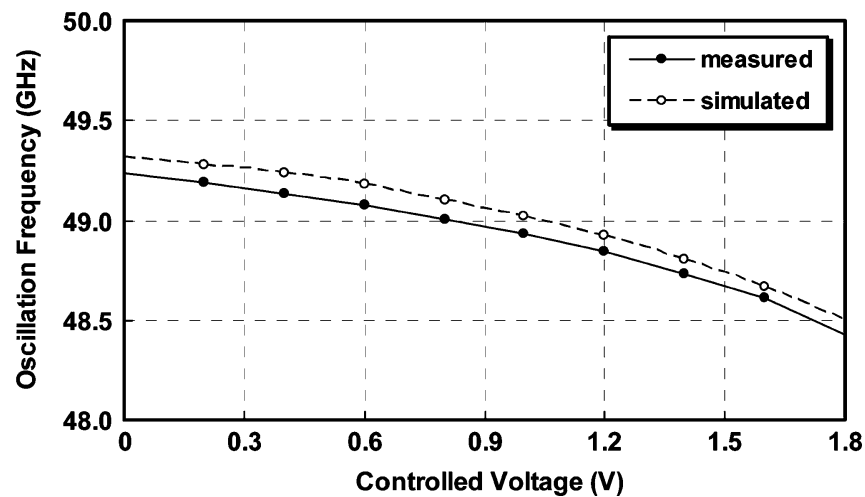

(a)

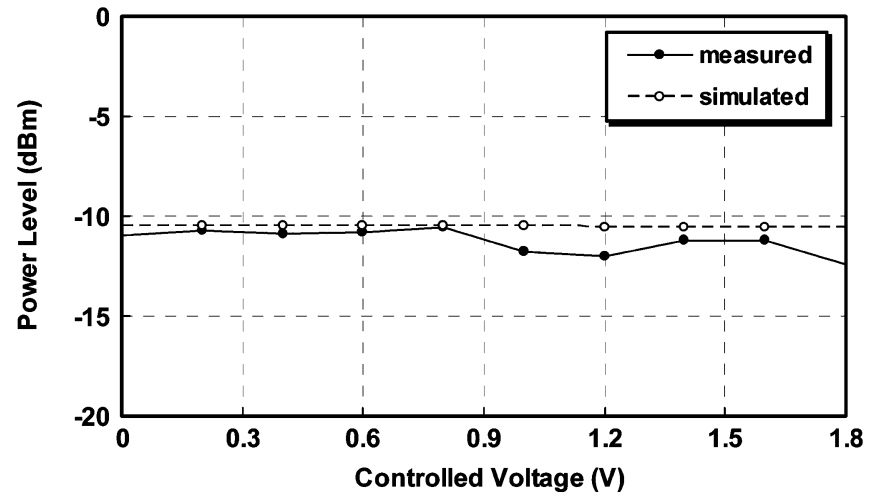

(b)

Fig. 14. (a) Oscillation frequency and (b) output power level of the VCO as the controlled voltage sweeps from 0 to $1.8 \mathrm{~V}$.

the VCO core can be minimized while maintaining sufficient output swing.

\section{CirCuit IMPlementation}

In the VCO design, it is desirable to reduce the shunt conductance for more relaxed startup conditions and enhanced phase noise. Due to the use of the admittance-transforming technique, the contribution from the varactor elements is thus minimized,

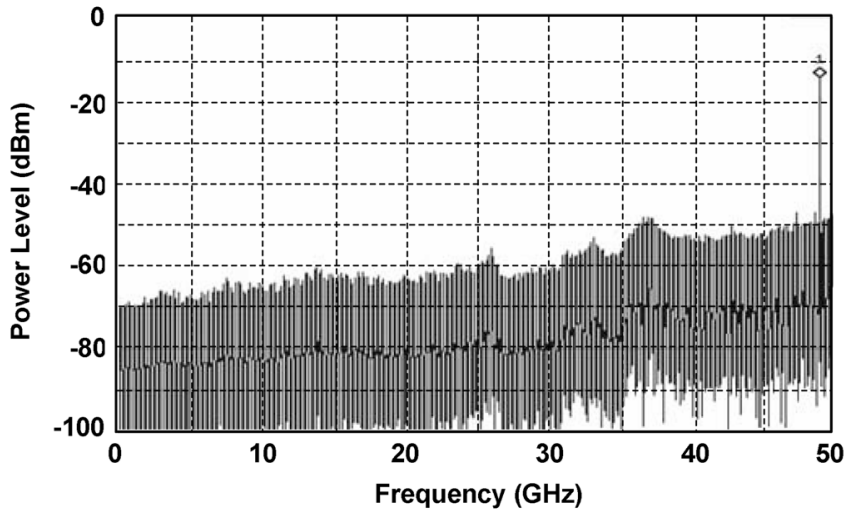

(a)

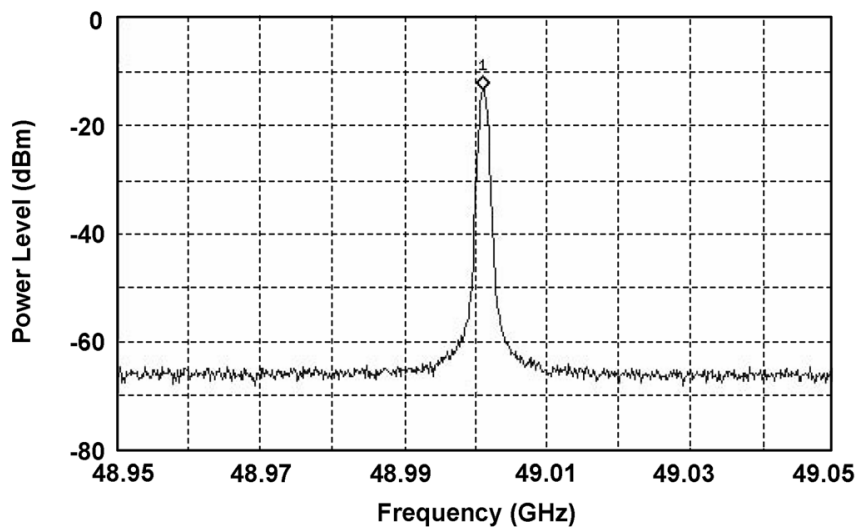

(b)

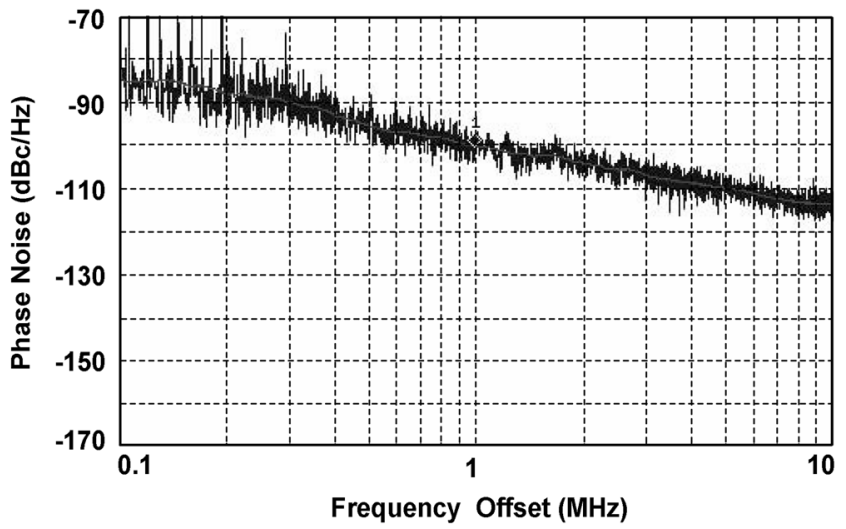

(c)

Fig. 15. Measured: (a) wideband spectrum, (b) narrowband spectrum, and (c) close-in phase noise of the fabricated VCO at $49 \mathrm{GHz}$.

especially at higher frequencies. Further performance improvement can be achieved by optimizing the $Q$ factors of the on-chip passive components.

In consideration of the $Q$ factor at millimeter-wave frequencies, the required inductors are implemented by the coplanar waveguide (CPW), which is constructed by one signal line surrounded by two adjacent grounded plates, as shown in Fig. 9. By properly choosing the dimensions of the linewidth $w$, the signal-to-ground spacing $s$, and the length $l$, the required inductance can be realized with sufficient accuracy. Based on the fullwave electromagnetic (EM) simulation, the extracted equivalent inductance and the $Q$ factor of the $\mathrm{CPW}$ at $50 \mathrm{GHz}$ are shown in 
TABLE II

PERFormance Summary of the CMOS VCOs AT THE 50-GHz FREQUENCY BAND

\begin{tabular}{|c|c|c|c|c|c|c|}
\hline & Unit & This Work & [2] & [5] & [14] & [9] \\
\hline Technology & - & $\begin{array}{c}0.18-\mu \mathrm{m} \\
\text { CMOS }\end{array}$ & $\begin{array}{c}0.18-\mu \mathrm{m} \\
\text { CMOS }\end{array}$ & $\begin{array}{c}0.18-\mu \mathrm{m} \\
\text { CMOS }\end{array}$ & $\begin{array}{c}0.18-\mu \mathrm{m} \\
\text { CMOS }\end{array}$ & $\begin{array}{c}0.12-\mu \mathrm{m} \\
\text { CMOS }\end{array}$ \\
\hline Frequency & $\mathrm{GHz}$ & 49 & 53 & 49 & 52 & 51 \\
\hline Tuning Range & $\mathrm{GHz}$ & \begin{tabular}{l|l}
0.8 & $1.1^{3}$ \\
\end{tabular} & 0.1 & $1.0^{2}$ & 0.6 & $1.0^{2,3}$ \\
\hline Phase Noise@1-MHz & $\mathrm{dBc} / \mathrm{Hz}$ & -101 & -86 & -96 & -97 & -85 \\
\hline $\mathrm{V}_{\mathrm{DD}}$ & $\mathrm{V}$ & 1.8 & - & 1.0 & 2.1 & 1.0 \\
\hline DC power & $\mathrm{mW}$ & 45 & 41 & 4 & 27 & 1 \\
\hline Output Power Level & $\mathrm{dBm}$ & $-11 \sim-12$ & -8 & $-26 \sim-27$ & -16 & -30 \\
\hline Phase & - & Differential & Single & Differential & Single & Differential \\
\hline $\mathrm{FOM}^{1}$ & $\mathrm{dBc} / \mathrm{Hz}$ & 178 & 164 & 184 & 177 & 179 \\
\hline
\end{tabular}

Fig. 10 for various $w$ and $s$ values. Note that, with the two separate ground plates in the CPW structure, underpasses using the second metal layer are also employed to suppress the unwanted odd-mode operations [12].

Another important design issue of the millimeter-wave VCO is the realization of the varactors. Fig. 11(a) shows the terminal connection of a conventional pMOS varactor where the body terminal is shorted to the source and the drain of the device. In this VCO design, varactors with the inversion-mode topology, as shown in Fig. 11(b), are employed to enhance the $Q$ factor at the higher frequency bands, while maintaining a relatively wide tuning range [13]. By connecting the body terminal to $V_{D D}$, the pMOS varactor can be operated in the accumulation region in addition to the strong, moderate, and weak inversion ones. As a result, the capacitance ratio of $C_{\mathrm{var}, \max } / C_{\mathrm{var}, \min }$ is effectively increased. Fig. 12 shows the simulated capacitance and $Q$ factor of the pMOS varactors at $50 \mathrm{GHz}$ for comparison.

Using a standard 1P6 M 0.18- $\mu \mathrm{m}$ CMOS process, a $U$-band $\mathrm{VCO}$ is implemented for demonstration. With a device layout optimized for the RF performance, the $n$-channel MOSFET in a deep n-well exhibits a maximum oscillation frequency $f_{\max }$ up to $60 \mathrm{GHz}$. As for the on-chip passive components, a top $\mathrm{AlCu}$ metallization layer with a thickness of $2 \mu \mathrm{m}$ and the metal-insulator-metal (MIM) structure with an oxide intermetal dielectric are provided in this technology. Table I summarizes the design parameters of the proposed VCO.

In order to verify the derivations from (10) to (12) in this circuit implementation, the values of $R_{s 1}, G_{\mathrm{var}}, G_{T}, L_{T}, G_{T}$, and $L \prime_{T}$ are calculated from Table I. The resulting values of $R_{s 1} G_{T}$ and $\left(\omega L_{T} / R_{s 1}\right)^{2}$ at the $50-\mathrm{GHz}$ frequency band are $3.14 \times 10^{-3}$ to $4.95 \times 10^{-3}$ and 68.0 to 92.7 , respectively, validating the assumptions of $R_{s 1} G_{T} \ll 1$ and $\left(\omega L_{T} / R_{s 1}\right)_{\gg}^{2} 1$ in the theoretical derivation. Meanwhile, by using the admittance-transforming technique, the shunt conductance $G_{\mathrm{var}}$ with an original value from 6.8 to $20.9 \mathrm{mS}$ is effectively suppressed to a value between $2.0-2.9 \mathrm{mS}$, leading to a relaxed startup condition for the $50-\mathrm{GHz}$ VCO design.

\section{EXPERIMENTAL RESULTS}

Fig. 13 shows the die photograph of the fabricated circuit with a chip area of $0.83 \times 0.87 \mathrm{~mm}^{2}$ including the RF pads. To evaluate the high-frequency performance, the output spectrum and phase noise were characterized by a $50-\mathrm{GHz}$ spectrum analyzer. The fabricated VCO was tested by on-wafer probing, while the losses from the measurement setup were calibrated and deembedded in the experimental results.

With a supply voltage of $1.8 \mathrm{~V}$, the VCO starts to oscillate at a dc current level of $1 \mathrm{~mA}$ due to the use of the admittance-transforming technique. In order to obtain sufficiently high output power, the performance of the $\mathrm{VCO}$ is characterized under a core bias current of $25 \mathrm{~mA}$. As the controlled voltage sweeps from 0 to $1.8 \mathrm{~V}$, the tuning characteristics, including the oscillation frequency and output power, are shown in Fig. 14. Within the output frequency range from 48.4 to $49.2 \mathrm{GHz}$, the $U$-band VCO exhibits an output power in the vicinity of $-11 \mathrm{dBm}$. By applying a controlled voltage up to $2.2 \mathrm{~V}$, an enhanced tuning range of $1.1 \mathrm{GHz}$ can be achieved. Fig. 15 shows the measured wideband and narrowband spectra and the close-in phase noise at $49 \mathrm{GHz}$, indicating a phase noise of $-100.6 \mathrm{dBc} / \mathrm{Hz}$ at $1-\mathrm{MHz}$ offset. Compared with the simulated result of $-100.9 \mathrm{dBc} / \mathrm{Hz}$ at $1-\mathrm{MHz}$ offset, good agreement is achieved due to accurate EM extraction. The performance of the VCO is summarized in Table II along with the results from previously published CMOS VCOs for comparison.

\section{CONCLUSION}

In this paper, an admittance-transforming technique has been presented for the design of the $L C$-tank CMOS VCOs at higher frequencies. By effectively reducing the shunt conductance contributed by the varactors, VCO circuits with an oscillation frequency close to the $f_{\max }$ of the active devices can be realized while maintaining the desirable circuit performance. Using a standard 0.18- $\mu \mathrm{m}$ CMOS technology, a $U$-band VCO based on the proposed technique is successfully demonstrated. It is well suited for low-cost implementations of the RF systems at millimeter-wave frequencies.

\section{ACKNOWLEDGMENT}

The authors would like to thank the National Chip Implementation Center, Hsinchu, Taiwan, R.O.C., for chip fabrication, and T.-P. Wang, Y.-C. Huang, and Prof. H. Wang, all with the National Taiwan University, Taipei, Taiwan, R.O.C., for chip measurement. 


\section{REFERENCES}

[1] B. Razavi, "A 60-GHz CMOS receiver front-end," IEEE J. Solid-State Circuits, vol. 41, no. 1, pp. 17-22, Jan. 2006.

[2] H. Shigematsu et al., "Millimeter-wave CMOS circuit design," IEEE Trans. Microw. Theory Tech., vol. 53, no. 2, pp. 472-477, Feb. 2005.

[3] C. H. Doan et al., "Millimeter-wave CMOS design," IEEE J. SolidState Circuits, vol. 40, no. 1, pp. 144-155, Jan. 2005.

[4] C. Cao and K. K. O, "Millimeter-wave voltage-controlled oscillators in 0.13- $\mu \mathrm{m}$ CMOS technology," IEEE J. Solid-State Circuits, vol. 41, no. 6, pp. 1297-1304, Jun. 2006.

[5] T.-N. Luo et al., "A 1-V CMOS VCO for 60-GHz applications," in IEEE Asia-Pacific Microw. Conf., Dec. 2005, vol. 1, 4 pp.

[6] J. Lee, J.-Y. Ding, and T.-Y. Cheng, "A 20-Gb/s 2-to-1 MUX and a 40-GHz VCO in $0.18 \mu \mathrm{m}$ CMOS technology," in VLSI Circuits Tech. Symp. Dig., Jun. 2005, pp. 136-139.

[7] A. P. van der Wel et al., "A robust 43-GHz VCO in CMOS for OC-768 SONET applications," IEEE J. Solid-State Circuits, vol. 39, no. 7, pp. 1159-1163, Jul. 2004

[8] F. Ellinger et al., "60 GHz VCO with wideband tuning range fabricated on VLSI SOI CMOS technology," in IEEE MTT-S Int. Microw. Symp. Dig., Jun. 2004, vol. 3, pp. 1329-1332.

[9] M. Tiebout, H. D. Wohlmuth, and W. Simburger, "A 1 V $51 \mathrm{GHz}$ fully integrated VCO in $0.12 \mu \mathrm{m}$ CMOS," in IEEE Int. Solid-State Circuits Conf. Tech. Dig., Feb. 2002, vol. 1, pp. 300-301.

[10] D. B. Leeson, "A simple model of feedback oscillator noise spectrum," Proc. IEEE, vol. 54, no. 2, pp. 329-330, Feb. 1966.

[11] A. W. L. Ng et al., "A 1-V 24-GHz 17.5-mW phase-locked loop in a $0.18 \mu \mathrm{m}$ CMOS process," IEEE J. Solid-State Circuits, vol. 41, no. 6, pp. 1236-1244, Jun. 2006.

[12] S.-C. Shin et al., "18-26 GHz low-noise amplifiers using 130 and 90-nm bulk CMOS technologies," in IEEE Radio Freq. Integrated Circuits Symp., Jun. 2005, pp. 47-50.

[13] P. Andreani and S. Mattisson, "On the use of MOS varactors in RF VCO's," IEEE J. Solid-State Circuits, vol. 35, no. 6, pp. 905-910, Jun. 2000.

[14] Y.-H. Cho et al., "A low phase noise 52-GHz push-push VCO in 0.18 $\mu \mathrm{m}$ bulk CMOS technologies," in IEEE Radio Freq. Integrated Circuits Symp., Jun. 2005, pp. 131-134.

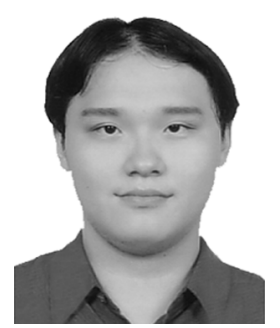

Hsieh-Hung Hsieh ( $\mathrm{S}^{\prime} 05$ ) was born in Taipei, Taiwan, R.O.C., in 1981. He received the B.S. degree in electrical engineering from National Taiwan University, Taipei, Taiwan, R.O.C., in 2004, and is currently working toward the Ph.D. degree in electronic engineering at National Taiwan University.

His research interests include the development of low-voltage and low-power RF integrated circuits, multiband wireless systems, RF testing, and monolithic microwave integrated circuit (MMIC) designs.

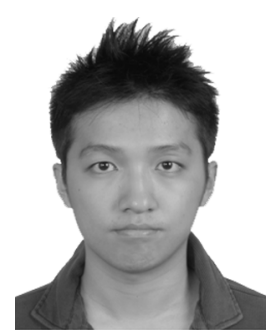

Yu-Hsin Chen (S'05-M'07) was born in Taipei, Taiwan, R.O.C., in 1983. He received the B.S. degree in electrical engineering from National Taiwan University, Taipei, Taiwan, R.O.C., in 2005 , and is currently working toward the M.S. degree in communication engineering at National Taiwan University.

His research interests include RF integrated circuits and monolithic microwave integrated circuit (MMIC) designs.

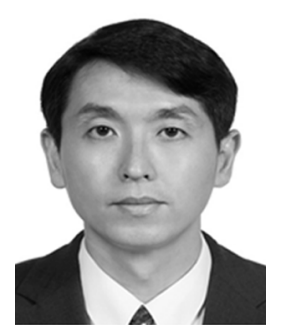

Liang-Hung Lu (M'02) was born in Taipei, Taiwan, R.O.C., in 1968. He received the B.S. and M.S degrees in electronics engineering from National Chiao-Tung University, Hsinchu, Taiwan, R.O.C., in 1991 and 1993, respectively, and the Ph.D. degree in electrical engineering from The University of Michigan at Ann Arbor, in 2001.

During his graduate study, he was involved in $\mathrm{SiGe}$ HBT technology and MMIC designs. From 2001 to 2002 , he was with IBM, where he was involved with low-power and RF integrated circuits for silicon-oninsulator (SOI) technology. In August 2002, he joined the faculty of the Graduate Institute of Electronics Engineering and the Department of Electrical Engineering, National Taiwan University, Taipei, Taiwan, R.O.C., where he is currently an Associate Professor. His research interests include CMOS/BiCMOS $\mathrm{RF}$ and mixed-signal integrated-circuit designs. 